\title{
Visible Meridian Phenomena after Acupuncture: A Series of Case Reports
}

\author{
Nikolay Dimitrov ${ }^{1}$, Nikola Tomov², Dimitrinka Atanasova ${ }^{1,3, *}$, Stiliyan lliev ${ }^{1}$, Tatyana Tomova ${ }^{4}$, \\ Dimitar Sivrev ${ }^{1}$, Zoya Goranova ${ }^{4}$ \\ ${ }^{1}$ Department of Anatomy, Faculty of Medicine, Trakia University, Stara Zagora, Bulgaria \\ 2Institute of Anatomy, University of Bern, Bern, Switzerland \\ 3/nstitute of Neurobiology, Bulgarian Academy of Sciences, Sofia, Bulgaria \\ ${ }^{4}$ Department Theory and Methodology of Kinesitherapy, National Sports Academy "Vassil Levski", Sofia, Bulgaria
}

Received March 20, 2020

Revised January 22, 2021

Accepted February 4, 2021

Correspondence to Dimitrinka Atanasova

Institute of Neurobiology, Bulgarian Academy of Sciences, Sofia, Bulgaria E-mail didiatanasova7@gmail.com
Background: In accordance with the meridian theory of Traditional Chinese Medicine (TCM), meridian phenomena are observed along the course of a meridian following acupuncture. Their visible manifestations include alterations in the color (reddening or whitening) of the skin as well as papule and vesicle formation.

Objectives: The aim of the present work is to report a series of visible meridian phenomena manifested in human subjects and to correlate them to TCM concepts. A total of 1,200 patients, on whom classical acupuncture (with standard single-use needles without electrostimulation or moxibustion) was applied, were carefully observed and documented.

Methods: Visible meridian phenomena were photographed using standard photographical equipment and compared to classical acupuncture channels used by TCM.

Results: Ten patients ( 5 male, 5 female) exhibited visible meridian phenomena. Lines, concurring with the meridians, were observed: white lines in seven cases and red lines in three cases. The duration of the two kinds of phenomena was different. White lines remained visible for a shorter period (10-15 min), whereas red lines were seen for up to one hour after needle removal.

Conclusion: These observations indicate that visible meridian phenomena following acupuncture are objective, albeit rare, findings that coincide with the acupuncture channels described in the classical works of TCM. The presence of such phenomena provides a new insight into the concept of meridians and explains the development of the idea in its historical context.

Keywords: Acupuncture, Meridian, Red line, White line

\section{INTRODUCTION}

Traditional Chinese Medicine (TCM) operates with a set of specific skin surface "points" which, upon connecting, form "channels" or "meridians" on the body surface. These meridians are the system of channels through which vital energy, or "Qi", flows through the body [1]. The traditional concept of the meridian theory is comprised of 12 standard channels, 12 collaterals, 8 extra meridians, 15 large collaterals, the musculature of 12 meridians, and 12 skin divisions. Meridians run on the surface of the whole body, both vertically and horizontally, integrating the inside with the outside [2].

However, meridians, as they were historically and traditio- nally developed, remain a vague concept. Multiple structures or regions of altered physical properties of the body have been considered to represent meridians, albeit without complete overlap. Additionally, some complex methods, certainly unavailable in antiquity, were proposed for meridian demonstration [1]. None of those methods or concepts gives a complete understanding of what the ancient Chinese physicians conceptualized as meridians.

The impression of meridian existence can be realized mainly in two aspects. The first is propagating needling sensations along the meridians (PNSAM) [3] -alternatively called propagated sensation along the channels (PSC) [4] - a kind of subjective propagating sensation, either spontaneously arising or induced by needling or other methods of stimulation [3]. 
The sensation patterns are, according to patient reports, mostly lines, zones, or slices. Their widths vary, both between subjects, meridians, and body parts, tending to be narrower at the limbs and wider on the trunk [3]. In the 1970s, the sensations along the meridians were investigated in a huge patient sample in China of which $\sim 0.3 \%$ of the subjects reported some sort of PNSAM [2]. Combining electrical and mechanical stimulation made it possible to trace out a line of feeling, coincident with the classical meridian course. Those experiments showed that the course of PNSAM propagation has higher electrical conductance [5]. The second aspect is the external appearance of objective changes either along or near the supposed meridian course on the body surface, including changes in skin color or the appearance of skin eruptions (macules, papules, and/or vesicles) [3]. It was suggested that some people (called meridian-sensitive subjects) can develop papuloid zones along the meridians upon stimulation [3]. In the present text, we will use the term "visible meridian phenomena" to denote any manifestations along putative meridians that are visible with the naked eye [6].

Many efforts have been devoted to prove the physical existence of meridians and their anatomic nature. The Korean physician, Bonghan Kim [7-9], announced the discovery of the meridian anatomy and named them "Bonghan ducts" or "primo ducts". Morphological studies related to Kim's theory have been conducted thereafter [10-13]. Putative acupuncture meridians in skin, skeletal muscle, and on organ surfaces were shown to get preferentially stained by vital dyes and to not coincide with blood or lymph vessels or nerves [14]. Furthermore, studies have noted the relationship between meridians and connective tissue. It was suggested that the connective tissue in areas along the meridians has certain special features $[15,16]$, probably related to the conductive properties of the interstitium [17].

It is also suspected that connective tissue resident mast cells are involved in the initiation and propagation of sensation and in the initiation of skin reddening by releasing vasoactive substances and causing hyperemia [18]. Moreover, mast cells tend to be distributed along the meridian line $[19,20]$. Infrared spectroscopy has shown the existence of "light channels" of high optical coherence in the human body, which follow along the meridian lines of TCM [21]. Needling-induced changes in body thermal images have also been observed. Higher temperature bands were seen running along channels on the extremities, especially when the patients felt PNSAM phenomena, giving an objective correlate to sensation [4].

All of those studies can be interpreted as providing indirect evidence for the existence of some morphofunctional peculiarities along meridian lines. However, despite all efforts, there is, to date, no undisputed evidence to show that acupuncture points or meridians exist as discrete entities [22].
The aim of the present work is to report multiple instances of visualization of meridian phenomena on the skin surface following acupuncture in human subjects. Observing visible meridian phenomena substantiates the concept of "meridians" and can explain the development of this complex idea in its historical context.

\section{MATERIALS AND METHODS}

The observations were conducted between 2013 and 2017 on 1,200 patients in the Via Vite Center for Eastern Medicine in Stara Zagora, Bulgaria; in the Experimental Laboratory of Chinese Medicine at the Faculty of Medicine in Stara Zagora, Bulgaria; at the Department of Theory and Methodology of Kinesitherapy, National Sports Academy, Sofia, Bulgaria; and in a private acupuncture practice in Sofia, Bulgaria. All of the patients gave their written informed consent to be included in the present study. Data handling was done in strict adherence to the principles of the Declaration of Helsinki. The majority of the patients underwent more than one procedure involving classical acupuncture, based on the TCM meridian theory and according to the symptoms at presentation, without additional electrostimulation and/or moxibustion.

The following standard single-use needles were used: $0.25 \times$ 50-mm, $0.30 \times 40$-mm, $0.30 \times 50$-mm stainless steel needles, (Beijing Zhongyan Taihe Medical Instrument Co., Ltd., China). Before insertion, the skin was briefly disinfected using a cotton swab soaked in $70 \%$ ethanol. The depth of insertion varied between subjects due to normal variation of skin and subcutaneous tissue thickness, but was usually between 0.5 and $1.5 \mathrm{~cm}$. Upon insertion, manual stimulation-lifting, thrusting, or rotating-was applied until a déqi sensation was reported by the patient. Needles were retained for up to 30 min, during which time they were controlled and revisited as appropriate. Details about the points needled are provided for each case.

Observed visible meridian phenomena were photographed by the respective professional performing acupuncture using standard photographical equipment available at the time and site of observation (Olympus PEN E-PL 1 camera and Samsung S3 and Samsung J5 smartphones) using default camera settings. The resulting images were not digitally altered in any way, apart from minor exposure and contrast correction and labeling. The observations were compared to a standard reference atlas of classical acupuncture channels used in TCM [23].

\section{RESULTS}

Among the 1,200 patients observed, 786 were female and 414 male; their age range was 6-85 years. Ten cases exhibited 
what was classified as visible meridian phenomena. Of those 10 cases, 7 (3 female, 4 male) demonstrated the formation of white lines and 3 ( 2 female, 1 male) the formation of red lines on the skin surface.

The width of the lines varied among the patients, ranging from a couple of millimeters to a couple of centimeters (most commonly between 0.5 and $1 \mathrm{~cm}$ ). The lines also had variable lengths-from $3-4 \mathrm{~cm}$ to $>20 \mathrm{~cm}$. Furthermore, they persisted for varying periods. White lines were observed over 10-15 min, before they faded and disappeared. Red lines persisted longer, for up to $1 \mathrm{~h}$, and remained visible even after needle removal.

In 9 out of 10 cases of visible meridian phenomena, the observed lines coincided with those used by TCM classical acupuncture meridians. One case could not be strictly interpreted in relation to a meridian passing through a stimulated acupoint. No correlation between subjective sensations reported by the patients and the appearance of visible meridian phenomena could be established.

\section{Visualization of white lines}

\section{1) Patient 1: visualization of white line coinciding with the bladder divergent meridian}

The white line phenomenon was observed in a 53-yearold female patient with disc herniations on several levels: L3L4, L4-L5, and L5-S1, presenting with long-standing low back pain and right leg pain. The patient responded well to acupuncture and reported lingering of symptoms after each needle application. On the third application, needles were placed in the following points: Bai'hui (GV20), Feng'chi (GB20), Fei'shu (BL13), Xin'shu (BL15), Ge'shu (BL17), Gan'shu (BL18), Pi'shu (BL20), Shen'shu (BL23), Zhi'shi (BL52), Da'chang'shu (BL25), Jia'ji (EX-B2, L3-L5), Da’zhui (GV14), Jian'jing (GB21), Ming'men (GV4), Ci'liao (BL32), Zhi'bian (BL54), Huan'tiao (GB30), Wei'zhong (BL40), and Kun'lun (BL60). After needle insertion, a white line was evident between Cheng'fu (BL36) and Wei'zhong (BL40) points (corresponding to Bladder meridian, medial branch - Fig. 1A) and remained visible for at least $10 \mathrm{~min}$, being $25-30 \mathrm{~cm}$ long and several millimeters thick (Fig. 1B). During presentation, the patient reported intensive feelings of paresthesia (de'qi) along the Bladder Divergent Meridian. After the procedure, the patient experienced a brief worsening of symptoms (interpreted as therapeutic crisis), followed by rapid and pronounced improvement.

\section{2) Patients 2 and 3: visualization of white line coinciding with Ren Mai meridian (Conception Vessel)}

This phenomenon was observed in two cases. The first one involved a 10-year-old boy suffering from asthma.

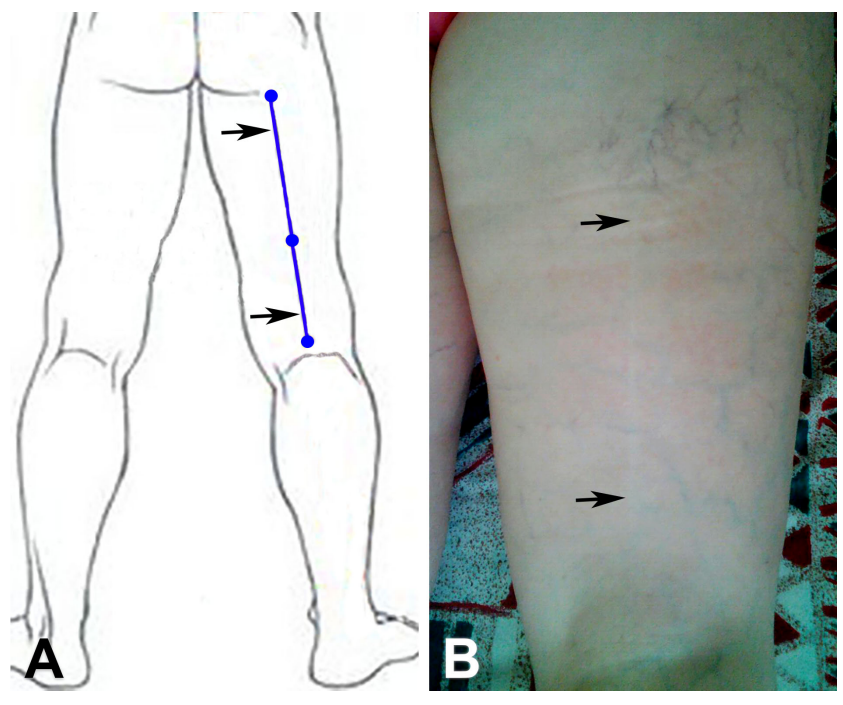

Fig. 1. Visible meridian phenomenon-white line. (A) The Bladder Divergent Meridian (blue line); (B) visible meridian phenomenon (arrows) between acupoints Cheng'fu (BL36) and Wei'zhong (BL40).

Needles were placed in Bai'hui (GV20), Yin'tang (EX-HN3), He'gu (LI4), Tai'xi (KI3), Nei'guan (PC6), Tai'yuan (LU9), and Dan'zhong (CV17) points. The line appeared between acupoint Dan'zhong (CV17) and Shen'que (CV8) shortly after needle placement and persisted for $\sim 15 \mathrm{~min}$. The width of the line was less than $5 \mathrm{~mm}$, the length $\sim 35 \mathrm{~cm}$, coinciding with the Ren Mai meridian (Fig. 2A, B).

The second case of a white line along the Ren Mai meridian was observed in the lower abdomen of a 35-year-old healthy male. The needles were placed in Qi'hai (CV6), Guan'yuan (CV4) points (Fig. 2C, D). The white line was seen briefly after needle placement, persisting for 10-15 min, between acupoint Qi'hai (CV6) and Guan'yuan (CV4), and was 5-6 cm long and several millimeters thick.

\section{3) Patient 4: visualization of white line coinciding with} Du mai meridian (Governing Vessel)

A white line coinciding with the Du Mai was visualized in a 55-year-old female with neck and back pain. The needles were placed in the following points: Feng'chi (GB20), Jian'jing (GB21), Chong'gu Zhui'dong (EX-HN), EX C5, A'shi'xue, Da’zhui (GV14), Ding'chuan (EX-B1), Tao'dao (GV13), EX Th2, Shen'zhu (GV12), EX Th4, Shen'dao (GV11), Feng'men (BL12), Fei'shu (BL13), Jue'yin'shu (BL14), and Xin'shu (BL15). The phenomenon appeared almost instantly after needling between acupoint Da'zhui (GV14) and Shen'dao (GV11) and remained visible for $20 \mathrm{~min}$. The white line was $23 \mathrm{~cm}$ long and up to $1 \mathrm{~cm}$ thick (Fig. 3B, C). This phenomenon can be associated with the Du Mai (Governing Vessel) (Fig. 3A). 


\section{4) Patients 5, 6, and 7}

Patient 5 was a 20 -year-old male with back pain. A white line appeared between Wei'shu (BL21) and Pi'shu (BL20) points ( $5 \mathrm{~cm}$ long), coinciding with the Bladder meridianthe medial branch, remaining visible for 10-15 min.

Patient 6 was a 40 -year-old male with back pain. A white line was seen between points Ge'shu (BL17), Du'shu (BL16), Xin'shu (BL15), and Jue'yin'shu (BL14) for up to $15 \mathrm{~min}$. It could be traced to the Bladder meridian-the medial branch.

Patient 7 was a 27-year-old, healthy male. A 2-3 cm-long white line, associated with the Hegu (LI4) point, was seen for

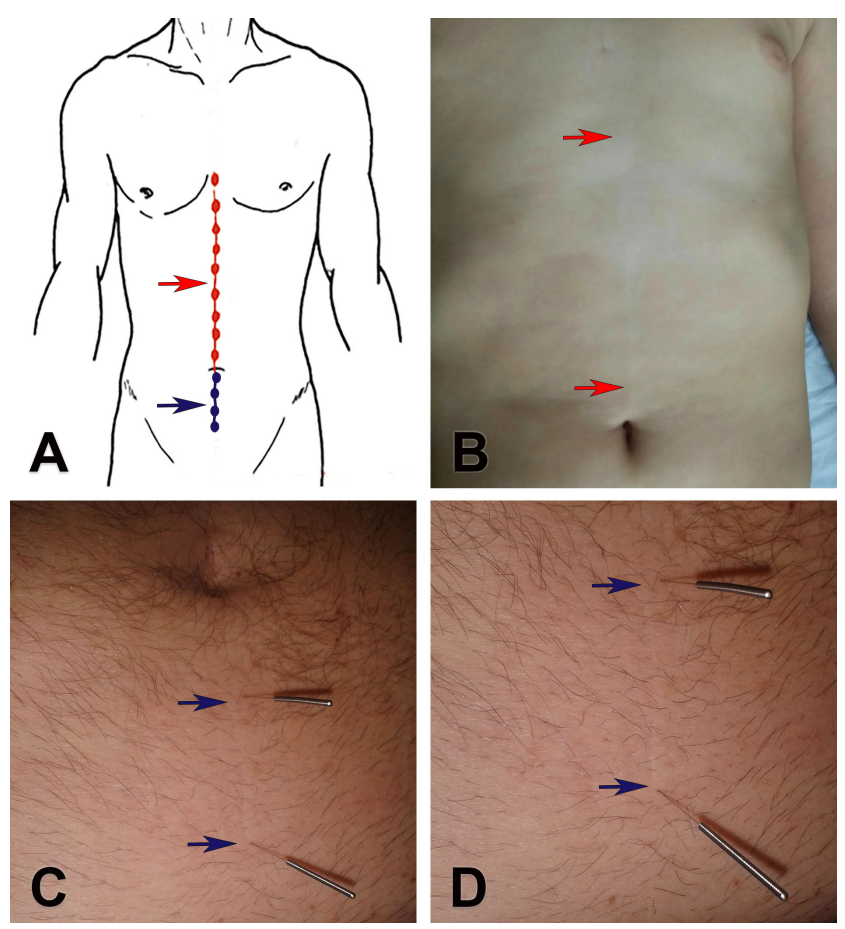

Fig. 2. Visible meridian phenomena-white line. (A) Ren Mai meridian (red arrow and red line; blue arrow and blue line). (B) Visible meridian phenomenon (red arrows) between acupoints Dan'zhong (CV17) and Shen'que (CV8). (C) and (D) Visible meridian phenomena (blue arrows) between acupoints Qi'hai (CV6) and Guan'yuan (CV4). $\sim 15 \mathrm{~min}$. However, this line did not follow the Large intestine meridian.

\section{Visualization of red lines}

\section{1) Patient 8: visualization of red line coinciding with the Du Mai meridian}

Red line formation was observed in a 65-year-old male with neck and back pain. The needles were placed in Feng'chi (GB20), Jian'jing (GB21), Chong'gu Zhui'dong (EX-HN), Da'zhui (GV14), and Shen'zhu (GV12) points. The phenomenon had a length of $\sim 10 \mathrm{~cm}$ and a thickness of $<5 \mathrm{~mm}$. It appeared after needling and was seen for $>60 \mathrm{~min}$ (Fig. 4). The red line followed the course of the Du Mai.

\section{2) Patient 9}

Patient 9 was a 55-year-old female with back pain. After needling of Kun'lun (BL60) point, the 3-5-cm-long red line followed the course of the Bladder divergent meridian, remaining visible for $>60 \mathrm{~min}$.

\section{3) Patient 10}

Patient 10 was a 45 -year-old female with neck and back pain. Needles were placed in the following points: Bai'hui (GV20), Fengchi (GB20), Fei'shu (BL13), Xin'shu (BL15), Ge'shu (BL17), Gan'shu (BL18), Pi'shu (BL20), Shen'shu (BL23), Zhi' shi (BL52), Da'chang'shu (BL25), Da'zhui (GV14), Jian'jing (GB21), Ming'men (GV4), Ci'liao (BL32), Zhi'bian (BL54), Huan'tiao (GB30), Cheng'fu (BL36), Yin'men (BL37), Wei'zhong (BL40), and Kun'lun (BL60). A several millimeters-thick red line between Xi'yang'guan (GB33) and Huan'tiao (GB30), following the Gallbladder meridian was seen and remained visible for $>20 \mathrm{~min}$.

\section{Summary of the results}

Visible meridian phenomena are extremely rare events, observed in only $0.83 \%$ of all observed subjects, on singular occasions.

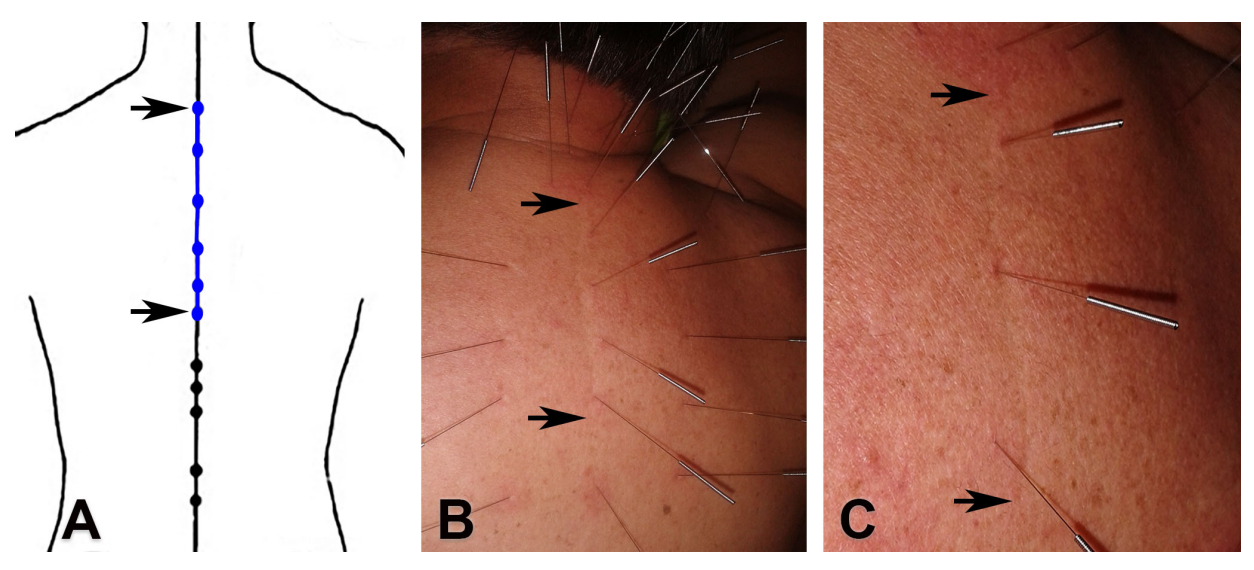

Fig. 3. Visible meridian phenomena-white line. (A) Du Mai merdian (arrows and blue line). (B) Visible meridian phenomenon (arrows) between acupoints Da'zhui (GV14) and Shen'dao (GV11). (C) Visible meridian phenomenon (arrows) between acupoints Da'zhui (GV14) and Shen'dao (GV11). Note the absence of similar lines between other needles in (B) and the approximately overlapping with the needling sites in (C). 


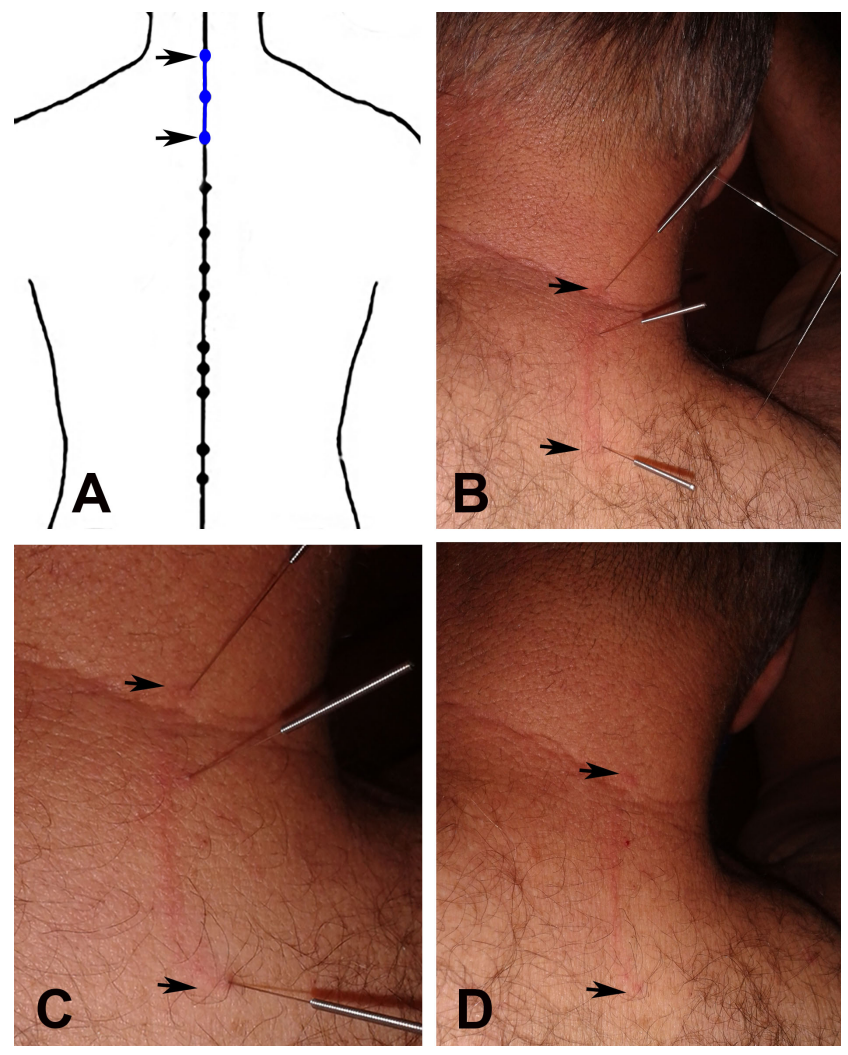

Fig. 4. Visible meridian phenomena-Red lines. (A) Du Mai meridian (arrows and blue line). (B) Visible meridian phenomenon (arrows) between acupoints Chong'gu Zhui'dong (EX-HN), Da'zhui (GV14), and Shen'zhu (GV12). (C) Visible meridian phenomenon (arrows) between acupoints Chong'gu Zhui'dong (EX-HN), Da'zhui (GV14), and Shen'zhu (GV12). (D) Visible meridian phenomenon (arrows) after needle removal between acupoints Chong'gu Zhui'dong (EX-HN) and Shen'zhu (GV12).

\section{Summary (of all observed subjects):}

$0.25 \%$ - exhibited red line phenomenon

$0.58 \%$ - exhibited white line phenomenon

$99.17 \%$ - exhibited no visible meridian phenomena

Visible meridian phenomena appeared rarely, only in $<1 \%$ of all observed subjects. Moreover, their appearance was a single event over the course of usually at least 10 acupuncture procedures. The frequency of white and red line appearance was different. White lines were more common (seen in $0.58 \%$ of subjects) than red lines (in $0.25 \%$ of subjects).

When present, visible meridian phenomena are easily recognizable and can be photographed without special equipment. Despite meticulous observation, we could not make a repeated observation of the visible meridian phenomenon in a subsequent procedure of the same patient.

As a general observation, it could be stated that white lines persisted for a shorter duration (10-15 $\mathrm{min}$ ) and disappeared before needle removal. The red lines were seen for longer periods, remaining visible for up to $1 \mathrm{~h}$ after the needles were removed.

\section{DISCUSSION}

Our observations indicate that visible meridian phenomena (white and red lines) exist; they are easily recognizable as such and can be documented without much effort. These visible meridian phenomena are a very rare occurrence, both in the patient cohort as well as during the course of multiple acupuncture procedures.

We suspect that the reason for the relative scarcity of reports is that they remain largely unnoticed by the practitioner, as they appear sometime after needle placement. It is highly likely that these visible meridian phenomena were observed by ancient Chinese physicians and were interpreted as what has been established to be meridians. The proof for this is the fact that the course of the observed white and red lines was identical with classical TCM channels in $90 \%$ of the cases. The cases in which the visible meridian phenomena do not coincide with classical channels could be correlated to the existence of collateral channels, which are also described [24].

The white and red lines are, to our judgment, two different phenomena. Their appearance is substantially different, and they remain visible for different duration (up to $15 \mathrm{~min}$ for white lines and $>1 \mathrm{~h}$ for red lines). Observations of skin reddening, similar to the lines in the present cases, have been reported previously. In meridian-sensitive subjects, "papuloid zones" (similar to urticarial manifestations) were observed along the meridians for 1-2 $\mathrm{h}$ after stimulation. They also coincided with propagating sensations and were almost consistent with the meridian courses recorded in ancient texts [3]. Our observation confirms the time frame of these phenomena, albeit without stimulation other than needling.

Visible or near-visible light phenomena cannot be excluded as contributing factors to the apparent image. Similar findings were made with highly specialized cameras. It has been postulated that meridians and acupoints could emit a strong "cold light", i.e., ultra-weak luminescence. High-intensity light-emitting points and lines were found on the body surface and completely coincided with the classical meridians and low electrical impedance lines [25]. Radiation field camera, point light vacuum imagers, and other equipment can also show supposed meridian phenomena in sensitive subjects [26]. Moreover, the optical properties of tissues are different between acupoints and non-acupoints [27].

A temperature response along meridians was reported, suggesting that meridians have infrared or near infrared radiation peculiarities. Acupuncture can induce hightemperature strips along meridians [28], which become more apparent when heating the acupoints or meridians [29]. Moreover, moxibustion (or similar infrared stimulation) of the body surface induces the appearance of "light channels", which presumably are identical to what are known as 
meridians [30].

Lines on the body surface can also be observed in the intact state, without additional stimulation $[31,32]$. Criticism of such observations on the body surface says that they are technical artifacts and are not identical to what are considered to be meridians. It was speculated that the visualization of energetic paths in the sense of meridians, as described in recent literature, is not possible using thermography, and that the reported thermographic image of meridians is due to physical-technical artifacts [33].

The anatomical basis of red visible meridian phenomena can be, at least partially, attributed to mast cells. Our previous work demonstrated the presence of mast cells in acupoints [34] and their degranulation following needling $[35,36]$, which can contribute to the erythema and reddening of the skin. Chemotactic migration of mast cells along the acupuncture meridians was also suspected [37].

The possible interaction between local cutaneous nerves and mast cells in the initiation of meridian phenomena is also noteworthy. Cutaneous nerve terminals respond to manual acupuncture with higher expression of substance $\mathrm{P}$ and calcitonin gene-related peptide, which is accompanied by aggregation of mast cells [38]. Aggregation and degranulation of histamine- and serotonin-containing mast cells can cause reddening and increased local blood flow in the acupoint [39]. The involvement of peripheral nerves is a widely known phenomenon called axon reflex, which is essentially a response to peripheral tissue injury [40]. The skin reddening seen at the site of the needle insertion is most probably a sign of local hyperemia. It can be caused by axon reflex through its direct action on cutaneous blood vessels and the local release of transmitters induced by the axon reflex [41]. Here, we can speculate that sensory nerve stimulation and the axon reflex are also involved in the propagating sensations along the meridians. Communication between adjacent nerve branches, leading to a robust axon reflex and modulation of afferent fiber transmission was previously shown to play a role in the production of acupuncture signals [40].

Sympathetic nerve endings in dermis are plexuses in arrector pili (AP) muscles. Therefore, it has been suggested that the propagation of sensation depends on the contraction of AP muscles and relies on sympathetic axon reflex through the reticular dermis/hypodermis [42]. Therefore, we suggest that the appearance of white lines on the skin is a phenomenon involving both sympathetically mediated contraction of blood vessels and action of AP muscles, pulling on skin connective tissue and mechanically compressing blood vessels passing through it. The same concept of the appearance of the white lines can be extended to connective tissue, as it is known to actively react to needle stimulation by contraction $[43,44]$.
Structures lying deeper than the skin might also be involved [45]. Recently, Benias et al. [46] described the existence of a previously undescribed interstitial network in the loose connective tissue. We believe that a partial explanation of the propagation of acupuncture signals can be found in this interstitial network, as it provides the means for easier movement ions, molecules, and/or cells in the direction of the meridian [17].

Despite our efforts, our attempts to replicate the appearance of visible meridian phenomena in the same patient were not successful; therefore, "meridian sensitivity" as a personal predisposition [3] could be rejected. However, the influence of factors unrelated directly to the acupuncture itself should be considered when looking for an explanation of the visible meridian phenomena. For instance, as 4 out of 10 observations were made before/during/after a thunderstorm, environmental factors should not to be ignored. Hyperemia around the needles might create optical contrast, making preexisting cicatrices, scratches, or other defects of the skin more visible (although in our observations, we did not establish such preexisting conditions). It should also be noted that the visual perception of multiple objects (red points around needle insertion sites) may lead to their unconscious combination in patterns or figures (pareidolia), which can be interpreted as channels by a biased acupuncturist.

At this stage of our research, we are unable to provide a robust explanation of the rarity of events, nor do we attempt to do so. The most practical reason could be that they are simply overlooked by the practitioner due to lack of meticulous observation or bad lighting conditions. Their appearance might well be only faint and invisible to an unaided eye. Furthermore, one could speculate about a correlation between the pathology of the organ and the appearance of visible meridian phenomena along the corresponding meridian. This is however unsubstantiated.

\section{CONCLUSIONS}

Visible meridian phenomena following acupuncture constitute an objective finding. They coincide with the acupuncture channels described in the classical works of TCM that were observed by ancient Chinese physicians. Their appearance is a very rare, but obvious event, which can be documented. It is highly possible that a number of physiological processes are simultaneously involved in what is seen as a single event-the appearance of visible lines on the body surface. The observation of similar phenomena without stimulation can lead to the impression that meridians might as well be natural occurrences made more obvious by acupuncture stimulation. Additional research is needed to clarify both the physiological mechanism as well as the 
morphological basis of the visible meridian phenomena.

\section{CONFLICT OF INTEREST}

The authors declare no conflict of interest.

\section{ORCID}

Nikolay Dimitrov, https://orcid.org/0000-0002-1016-9788

Nikola Tomov, https://orcid.org/0000-0002-7072-3832

Dimitrinka Atanasova,

https://orcid.org/0000-0003-1745-7755

Stiliyan Iliev, https://orcid.org/0000-0003-1289-7153

Tatyana Tomova, https://orcid.org/0000-0002-2394-2979

Dimitar Sivrev, https://orcid.org/0000-0002-2134-9522

Zoya Goranova, https://orcid.org/0000-0002-5508-4980

\section{REFERENCES}

1. Longhurst JC. Defining meridians: a modern basis of understanding. J Acupunct Meridian Stud 2010;3:67-74.

2. Wang GJ, Ayati MH, Zhang WB. Meridian studies in China: a systematic review. J Acupunct Meridian Stud 2010;3:1-9.

3. Jin GY, Jin JJX, Jin LL. Contemporary studies of the meridian phenomena. In: Jin GY, Jin JJX, Jin LL, eds. Contemporary Medical Acupuncture - a Systems Approach. New York: Springer, 2007:44-8.

4. Liu R, Zhuang D, Yang X, Li Y, Zhang D, Wen B, et al. [Objective display on phenomena of propagated sensation along channels (PSC)--changes on the infrared thermal image channels pathway of upper extremity]. Zhen Ci Yan Jiu 1990;15:239-44. Chinese.

5. Zhu ZX, Yan ZQ, Yu SZ, Zhang RX, Wang JY, Liu YM, et al. Studies on the phenomenon of latent propagated sensation along the channels. I. The discovery of a latent PSC and a preliminary study of its skin electrical conductance. Am J Chin Med 1981;9:216-24.

6. Zhang W, Wang Z, Jia S, Tian Y, Wang G, Li H, et al. Is there volume transmission along extracellular fluid pathways corresponding to the acupuncture meridians? J Acupunct Meridian Stud 2017;10:5-19.

7. Kim BH. [Study on the reality of acupuncture meridians]. J Jo Sun Med 1962;9:5-13. Korean.

8. Kim BH. On the Kyungrak system. J Acad Med Sci DPR Korea 1963;90:1-41.

9. Kim BH. [Sanal theory]. J Jo Sun Med 1965;108:39-62. Korean.

10. Baik KY, Lee JW, Lee BC, Johng HM, Nam TJ, Sung B, et al. Acupuncture meridian and intravascular Bonghan duct. Key Eng Mater 2005;277-279:125-9.

11. Baik KY, Ogay V, Jeoung SC, Soh KS. Visualization of Bonghan microcells by electron and atomic force microscopy. J Acupunct
Meridian Stud 2009;2:124-9.

12. Lee BC, Yoo JS, Baik KY, Kim KW, Soh KS. Novel threadlike structures (Bonghan ducts) inside lymphatic vessels of rabbits visualized with a Janus Green B staining method. Anat Rec B New Anat 2005;286:1-7.

13. Fujiwara S, Yu SB. ["Bonghan theory" morphological studies]. Igaku no Ayumi 1967;60:567-77. Japanese.

14. Lee BC, Soh KS. Visualization of acupuncture meridians in the hypodermis of rat using Trypan blue. J Acupunct Meridian Stud 2010;3:49-52.

15. Xu C, Zheng L. Present status of the relationship between meridians and connective tissues. Shanghai J Acupunct 2003;22: 45-9.

16. Zheng L. Channels may be functional zones adhering to certain tissues. Zhongguo Zhen Jiu 2001;21:35-6.

17. Tomov N, Atanasova D, Dimitrov N. Is the newly described interstitial network the anatomical basis of acupuncture meridians? A commentary. Anat Rec (Hoboken) 2020;303:216970 .

18. Song J. [Mast cell and meridian phenomenon]. Liaoning J Tradit Chin Med 1977;4:59-61. Chinese.

19. Lin J, Ren Z, Yan A, Wu J, Li J, Chang G. [Observation of mast cells from connective tissue in human acupoint by morphological methods and immunohistochemistry]. Henan Tradit Chin Med 1989;9:2-6. Chinese.

20. Zhu Z, Xu R. [Morphometric observation on the mast cells under the acupuncture meridian lines]. Zhen Ci Yan Jiu 1990;15: 157-8. Chinese.

21. Popp FA, Maric-Oehler W, Schlebusch KP, Klimek W. Evidence of light piping (Meridian-Like Channels) in the human body and nonlocal EMF effects. Electromagn Biol Med 2009;24:35974.

22. Ramey DW. Acupuncture points and meridians do not exist. Sci Rev Altern Med 2001;5:143-8.

23. Focks C. Atlas of Acupuncture. Edinburgh: Churchill Livingstone - Elsevier, 2008. 732 p.

24. Zhang WB, Wang GJ, Fuxe K. Classic and modern meridian studies: a review of low hydraulic resistance channels along meridians and their relevance for therapeutic effects in traditional Chinese medicine. Evid Based Complement Alternat Med 2015;2015:410979.

25. Yan Z, Yu S. [Exploration on the relationship between propagated sensation along channels and variation in luminescence of acupuncture points]. J Tradit Chin Med 1980;8:53-6. Chinese.

26. Lin X, Lu W, Li G, Guan Y. A research into electro-luminescence of meridians and acupuncture points on human bodies. Discov Nat 1990;9:50-2.

27. Li L, Yang HQ, Xie SS. Meridian optics: a photonics research on meridian phenomena. J Sci Chin 2007;37:62-7.

28. Zhang D, Fu WX, Wang SY, Ma HM, Wang YC. [Comparison of high temperature lines along the meridians by different 
acupuncture methods]. Zhongguo Zhen Jiu 2000;20:29-33. Chinese.

29. Hu XL, Xu JS, Ye L, Yang J, Wang PQ, Wu BH. Elicitation of infrared radiant track along meridian courses over human body surface by local heating. J Infrared Millim Waves 2002;21:6-8.

30. Schlebusch KP, Maric-Oehler W, Popp FA. Biophotonics in the infrared spectral range reveal acupuncture meridian structure of the body. J Altern Complement Med 2005;11:171-3.

31. Hu X, Wu B, Wang P. [Displaying of meridian courses travelling over human body surface under natural conditions]. Zhen Ci Yan Jiu 1993;18:83-9. Chinese.

32. Wang $\mathrm{P}, \mathrm{Hu} \mathrm{X}, \mathrm{Wu}$ B. [Displaying of the infrared radiant track along meridians on the back of human body]. Zhen Ci Yan Jiu 1993;18:90-3, 89. Chinese.

33. Litscher G. Infrared thermography fails to visualize stimulationinduced meridian-like structures. Biomed Eng Online 2005;4: 38.

34. Dimitrov N, Atanasova D, Tomov N, Ivanova I, Staykova Y, Dinkova K, et al. Distribution of histamine-positive mast cells in the vicinity of the needle tract following acupuncture in "Zusanli" (ST36) acupoint in rats. Acta Morphol Anthropol 2016;23:26-31.

35. Dimitrov N. Morphological changes in biologically active point /BAP/ ST36 after acupuncture in rat. Acta Morphol Anthropol 2012;19:30-3.

36. Dimitrov N, Atanasova D, Tomov N, Sivrev D, Lazarov N. Acupuncture causes serotonin release by mast cells. Rom J Morphol Embryol 2017;58:961-8.

37. Yang YM, Waug PP. [Morphological observation on the effect of acupuncture on mast cells at the Zusanli point]. Zhen Ci Yan Jiu
1986;11:298-302. Chinese.

38. Wu ML, Xu DS, Bai WZ, Cui JJ, Shu HM, He W, et al. Local cutaneous nerve terminal and mast cell responses to manual acupuncture in acupoint LI4 area of the rats. J Chem Neuroanat 2015;68:14-21.

39. Cheng KJ. Neurobiological mechanisms of acupuncture for some common illnesses: a clinician's perspective. J Acupunct Meridian Stud 2014;7:105-14.

40. Zhang ZJ, Wang XM, McAlonan GM. Neural acupuncture unit: a new concept for interpreting effects and mechanisms of acupuncture. Evid Based Complement Alternat Med 2012;2012: 429412.

41. Yaprak M. The axon reflex. Neuroanatomy 2008;7:17-19.

42. Liu L, Pan J, Zhang H. Study on the pilomotor line and its mechanism of sympathetic axon reflex for the propagation of acupuncture signals. Chin J Basic Med Tradit Chin Med 2004;2: 50-5.

43. Langevin HM, Bouffard NA, Badger GJ, Churchill DL, Howe AK. Subcutaneous tissue fibroblast cytoskeletal remodeling induced by acupuncture: evidence for a mechanotransductionbased mechanism. J Cell Physiol 2006;207:767-74.

44. Langevin HM. Acupuncture, connective tissue, and peripheral sensory modulation. Crit Rev Eukaryot Gene Expr 2014;24:24953.

45. Langevin HM, Yandow JA. Relationship of acupuncture points and meridians to connective tissue planes. Anat Rec 2002;269: 257-65.

46. Benias PC, Wells RG, Sackey-Aboagye B, Klavan H, Reidy J, Buonocore D, et al. Structure and distribution of an unrecognized interstitium in human tissues. Sci Rep 2018;8:4947. 